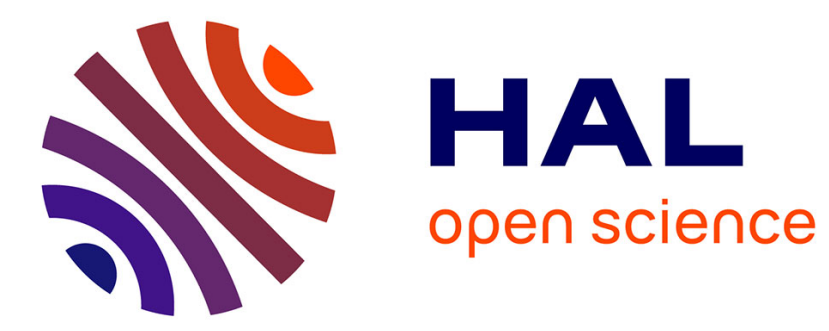

\title{
Theoretical and experimental studies of critical microemulsions
}

\author{
P. Tartaglia, E. Ducros, P. Chabrat, J. Rouch, Si Chen
}

\section{To cite this version:}

P. Tartaglia, E. Ducros, P. Chabrat, J. Rouch, Si Chen. Theoretical and experimental studies of critical microemulsions. Journal de Physique IV Proceedings, 1993, 03 (C1), pp.C1-249-C1-257. 10.1051/jp4:1993121 . jpa-00251564

\section{HAL Id: jpa-00251564 https://hal.science/jpa-00251564}

Submitted on 1 Jan 1993

HAL is a multi-disciplinary open access archive for the deposit and dissemination of scientific research documents, whether they are published or not. The documents may come from teaching and research institutions in France or abroad, or from public or private research centers.
L'archive ouverte pluridisciplinaire HAL, est destinée au dépôt et à la diffusion de documents scientifiques de niveau recherche, publiés ou non, émanant des établissements d'enseignement et de recherche français ou étrangers, des laboratoires publics ou privés. 


\title{
Theoretical and experimental studies of critical microemulsions
}

\author{
P. TARTAGLIA, E. DUCROS ${ }^{*}$, P. CHABRAT* ${ }^{*}$ J. ROUCH ${ }^{*}$ and S.H. CHEN**
}

Dipartimento di Fisica, Università di Roma La Sapienza, Piazzale Aldo Moro 2, 00185 Roma, Italy

* Centre de Physique Moléculaire Optique et Hertzienne, URA 283 du CNRS, Université Bordeaux I, 351 Cours de la Liberation, 33405 Talence cedex 5, France

** Department of Nuclear Engineering and Center for Materials Science and Engineering, Massachusetts Institute of Technology, Cambridge, MA 02139, U.S.A.

ABSTRACT. We report on the extension of the droplet model to account for static and dynamic light scattering studies of a ternary water-in-oil microemulsion close to its critical point. This model, which assumes that the critical microemulsion droplets are forming transient polydisperse fractal aggregates, reproduces well the usual features of critical phenomena and is also able to account, in a very natural way, for the dynamical background effects evidenced experimentaly.

Le modèle des gouttelettes a été étendu pour rendre compte de la diffusion statique et dinamique de la lumière par une microemulsion critique ternaire eau dans huile. En supposant que les gouttelettes forment des agrégats polydispersés de nature fractale, on peut rendre compte avec une excellente précision des phénomènes observés. En particulier on dêcrit les effets du fond dynamique mis en évidence expérimentalement. 
Extensive theoretical /1,2/ and experimental /3/ literature on critical phenomena in fluids has been accumulated in recent years. An important consequence of these efforts has been the realization that sufficiently close to the critical point, all critical phenomena in simple fluids (liquid-gas critical point) as well as in multicomponent fluids (consolute critical point) share universal features in terms of having identical critical exponents and the static and dynamic scaling functions. Universal critical indices governing the divergence of the compressibility and of the long range correlation length, respectively $\gamma=1.24$ and $v=0.63$, are those corresponding to the same universality class i.e. a three dimensional system having a scalar order parameter, like simple fluids or binary systems at constant pressure. In the case of a simple fluid, the density is the order parameter, whereas in the case of binary systems, this role is played by the concentration. For simple fluids, like xenon or molecular binary or ternary mixtures such as nitrobenzene- $n$-hexane or water-benzene-ethanol, the value of the short range correlation length which is similar to the range of the interatomic potential, is of the order of a few Angstroms /4, 5/. For supramolecular fluids like a critical micellar solution of ethylene glycol mono-n-dodecyl ether $\left(\mathrm{C}_{12} \mathrm{E}_{5}-\mathrm{H}_{2} \mathrm{O}\right)$ and water, the diameter of the micelles is of the order of $200 \AA / 6$ / which is ten times greater than the range of the interatomic potential among the constituting amphiphilic molecules. The situation is similar for a critical AOT-water-decane microemulsion system where the radius of the microemulsion droplets is of the order of $80 \AA$. It is therefore quite likely that the macroscopic size of the aggregates strongly influences the critical behavior of supramolecular fluid in many different ways in particular through the occurence of strong dynamical background effects $/ 7 /$.

The aim of this paper is to present new preliminary results deduced from the so-called dynamical droplet model which extends previous works of Martin et al. /8/. Our theoretical results will then be compared to extensive experimental data we obtained in the critical regime of an AOT water-in-oil microemulsions. A particular emphasis will be devoted to the behavior of the scattered intensity and to the first cumulant, which reflects the critical slowing down of the density-density correlation function, as deduced from quasi-elastic light scattering measurements. As we shall see, a good agreement is obtained between the model and the experiments even in the crossover regime which was previously not well accounted for by mode coupling or mode decoupling theories $/ 9 /$. 
The static and dynamic scattering properties of the critical microemulsion system can be derived interpreting the correlated regions of the critical fluctuations of the order parameters as physical clusters. The calculation can then be performed along the lines proposed by Martin et al $18 /$, and Teixeira and Chen $/ 10 /$ for percolative clusters and colloidal fractal aggregates. We have applied the same approac $h$ to the study of the percolative behavior of the same microemulsion system /11/. For spherical dielectric spheres in the Rayleigh-Gans regime, the scattered intensity at scattering wave vector $\mathrm{q}, \mathrm{I}(\mathrm{q})$ reads

$$
I(q)=N_{p} P(q) S(q)
$$

$\mathrm{N}_{\mathrm{p}}$ is the number of scattering particles per unit volume, $\mathrm{S}(\mathrm{q})$ is the static structure factor and accounts for interactions among the droplets forming a cluster. $P(q)$ is the form factor which depends on the radius $R_{1}$ of the monomer assumed to be spherical. In the case of the critical AOT/Water/Decane microemulsion system at molar ratio $\mathrm{X}$ of water to surfactant equal to 40.8 . We assume the system to be made of water-in-oil droplets; it has been shown by neutron and light scattering that the radius of individual droplets is close to $85 \AA$. Accounting for the values of the wave vector $q$ in a standard quasi-elastic light scattering experiment we obtain for $\mathrm{qR}_{1}$ a value much smaller than one. Thus the form factor can be put equal to unity with a very good accuracy. To within a proportionality constant, the intensity scattered by a cluster consisting of $\mathrm{k}$ particles is given by the static structure factor $\mathrm{S}_{\mathrm{k}}(\mathrm{q})$ which is

$$
S_{k}(q)=\int d r \exp (i \vec{q} \vec{r}) g_{k}(r)
$$

where $g(r)$ is the pair distribution function for an object of fractal dimension $\mathrm{D}$

$$
g_{k}(r)=\frac{D}{4 \pi} \frac{1}{R_{1}^{D}} r^{D-3} \exp \left[-r / R_{k}\right]
$$

In eq (11) $R_{k}$ is a cutoff parameter which has the physical meaning of average size of the cluster,. $R_{k}$ and $R_{1}$ being connected by

$$
R_{k}=R_{1} k^{1 / D}
$$

By combining eqs (2) and (3) it is straightforward to calculate $S_{k}(q)$ 


$$
S_{k}(q)=k \frac{\sin \left[(D-1) \arctan \left(q R_{k}\right)\right]}{(D-1) q R_{k}\left(1+q^{2} R_{k}^{2}\right)^{\frac{D-1}{2}}}
$$

that will be approximated in our case as

$$
S_{k}(q) \approx k \exp \left[-\frac{D(D+1)}{6} q^{2} R_{k}^{2}\right]
$$

Recently the interpretation of critical fluctuations in terms of fractal clusters has been formulated precisely from the theoretical point of view $/ 11 /$ and gives the fractal dimension $D$ of the cluster as $D=d-\beta / v$ where $\beta$ and $v$ are respectively the index of the coesistence curve and of the long range correlation length and $d=3$ is the dimensionality of space. The hypothesis has also been verified experimentally in the case of a binary mixture $/ 12 /$. Using the critical scaling law $\beta / v=(d-2+\eta) / 2$, where $\eta$ is the Fisher exponent for the critical correlation function, we get a value of $D=(5-\eta) / 2$ for the fractal dimension, very close to that obtained for percolation. Moreover $\mathrm{D}$ and the index $\tau$ connected to the distribution of clusters, as we will see shortly, are linked through the hyperscaling relation $D(\tau-1)=d$ leading to $\tau=(11-\eta) /(5-\eta)$ a value also close to that obtained for percolation clusters. In fact, in analogy with percolating clusters, we will assume that the cluster size distribution function $\mathrm{N}(\mathrm{k})$ for critical aggregates scales according to the law $N(k) \approx k^{-\tau} f\left((k / s)^{\sigma}\right) / 14 /$. The parameter $s$ has the meaning of the average number of monomers per cluster, $\sigma \approx 0.5$ and $\tau$, the polydispersity index, are universal exponents. The cutoff function $f(x)$ is assumed to be a Gaussian, then accounting for the numerical value of $\sigma$, the normalized cluster size distribution function becomes

$$
\mathrm{N}(\mathrm{k})=\frac{\mathrm{s}-2}{\Gamma\left(2-\mathrm{t}, \frac{1}{\mathrm{~s}}\right)} \mathrm{k}^{-\tau} \exp \left[-\frac{\mathrm{k}}{\mathrm{s}}\right]
$$

where $\Gamma(x, y)$ is the incomplete Euler Gamma function.

The total intensity I(q) scattered by the collection of the clusters is the incoherent sum of the intensity scattered by each of them

$$
I(q)=\int_{1}^{\infty} d k k N(k) S_{k}(q)
$$


and can be calculated explicitely. One can then define scaling variable $\mathrm{x}=\mathrm{q} \xi$, where $\xi$ is the correlation length, which can be expressed in term of the dimension of the average size cluster by $\xi=h_{1} s^{1 / D}$, with $h=[D(D+1) / 6]^{1 / 2}$, and derive for the intensity

$$
\mathrm{I}(\mathrm{x})=\Gamma(\mathrm{D}+1)\left[\frac{\xi}{\mathrm{hR_{1 }}}\right]^{\mathrm{D}} \frac{\Gamma\left(3-\tau,\left[\frac{\xi}{\mathrm{hR}}\right]^{-\mathrm{D}}\left(1+\mathrm{x}^{2}\right)^{\frac{\mathrm{D}}{2}}\right)}{\Gamma\left(2-\tau,\left[\frac{\xi}{\mathrm{hR}}\right]_{1}^{-\mathrm{D}}\left(1+\mathrm{x}^{2}\right)^{\frac{\mathrm{D}}{2}}\right)}\left(1+\mathrm{x}^{2}\right)^{-\frac{\mathrm{D}(3-\tau)}{2}}
$$

From the same model we can also deduce the dynamical behavior of the critical system by calculating the first cumulant $\Gamma_{c}$ of time dependent density-density correlation function $G(q, t)$ which is given by $\Gamma_{\mathrm{c}}=[\mathrm{d} \ln \mathrm{G}(\mathrm{t}) / \mathrm{dt}]_{\mathrm{t}=0 .}$. Assuming the rigidity of the fractal clusters. $\Gamma_{\mathrm{c}}$ can be expressed by

$$
\Gamma_{c}(x)=\frac{q^{2}}{I(x)} \int_{1}^{\infty} d k k N(k) S_{k}(q) D_{k}
$$

where $D_{k}$, the translational diffusion coefficient of the k-cluster, is simply connected to $D_{1}$ the diffusion coefficient of the monomer droplet by $D_{k}=D_{1} k^{-1 / D}$. From eq (10) and using the same method outlined above, it is possible to infer the scaled first cumulant $\Gamma_{c}^{*}=\Gamma_{c} /\left(D_{1} R_{1} q^{3}\right)$

$$
\Gamma_{\mathrm{c}}^{*}(\mathrm{x})=\frac{\Gamma\left(3-\tau-\frac{1}{\mathrm{D}} \cdot\left[\frac{\xi}{\mathrm{hR}}\right]^{-\mathrm{D}}\left(1+\mathrm{x}^{2}\right)^{\frac{\mathrm{D}}{2}}\right)}{\Gamma\left(3-\tau,\left[\frac{\xi}{\mathrm{hR}}\right]_{1}^{-\mathrm{D}}\left(1+\mathrm{x}^{2}\right)^{\frac{\mathrm{D}}{2}}\right)} \frac{\mathrm{h}}{\mathrm{x}}\left(1+\mathrm{x}^{2}\right)^{\frac{1}{2}}
$$

In the case of mode coupling theory of critical phenomena, the scattered intensity and the order parameter relaxation rate $\Gamma_{c}^{*}$ are scaling functions of the variable $\mathbf{x}$. I(q) obeys Ornstein-Zernike relation whereas $\Gamma_{\mathbf{c}}{ }^{*}$ is given by the well known Kawasaki's formula /15/. In the present model I(q) and $\Gamma_{c}{ }^{*}$ given above are function not only of the scaling variable $x$ but also of the ratio $\xi / R_{1}$ which explicitily depends on the size of the monomers, bearing a priori striking differences with Kawasaki mode coupling /15/ or Perl and Ferrel /16/ mode decoupling results.

Let us discuss now in more detail the specific case of microemulsions formed in a ternary solution of AOT/Water/Decane. The phase diagram of the mixture is extremely sensitive to the ratio $w$ and to the volume fraction $\phi$ of the dispersed phase. $\phi$ is defined as usually by assuming the volumes 
additivity i.e. $\phi=\left(\mathrm{V}_{\mathrm{H}_{2} \mathrm{O}}+\mathrm{V}_{\mathrm{AOT}}\right) /\left(\mathrm{V}_{\text {Decane }}+\mathrm{V}_{\mathrm{H}_{2} \mathrm{O}}+\mathrm{V}_{\mathrm{AOT}}\right)$. On the phase diagram reported on Fig. 1 and corresponding to $X=40.8$, one observes a lower critical consolute point located at a volume fraction of the dispersed phase $\phi_{c}=0.098$ and at a temperature $T_{c}=39.98^{\circ} \mathrm{C}$. The system shows also a rather extended percolation line and a wide region characterized by a lamellar phase.

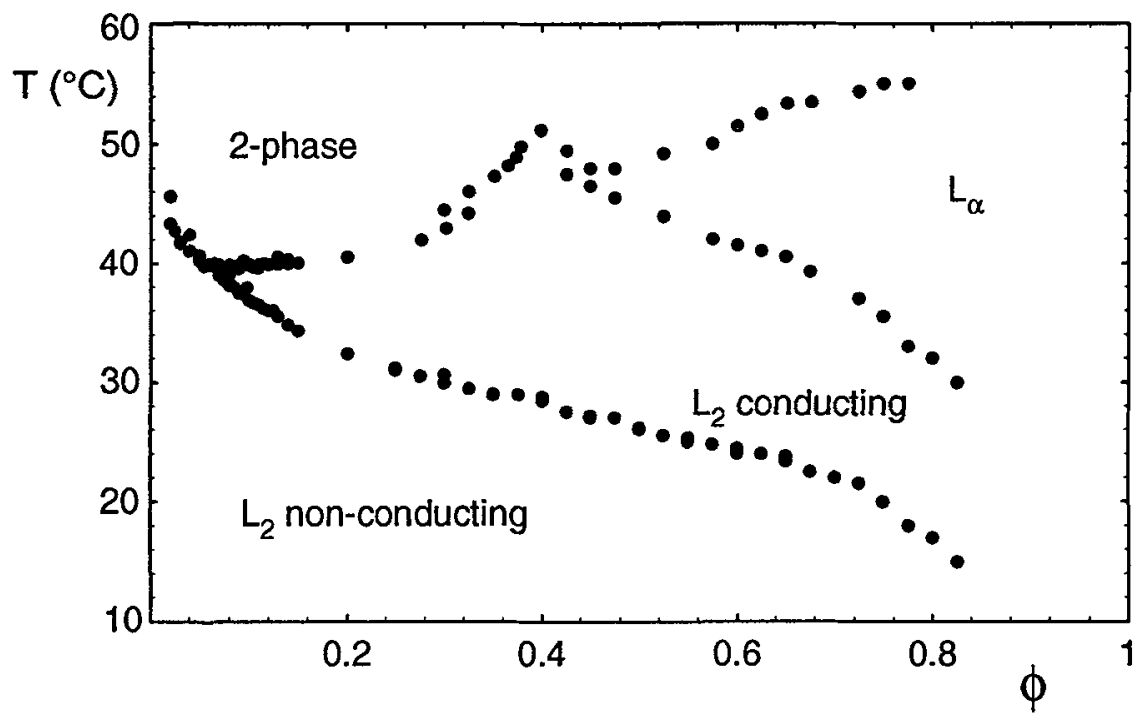

Fig. I The phase diagram of the microemulsion AOT/Water/Decane, showing the two- and one-phase regions, the percolation line which separates the conducting and non-conducting one-phase $\mathrm{L}_{2}$ regions, and the lamellar phase $L_{\alpha}$.

The experimental results $/ 9 /$ for the scattered intensity at the critical composition, as a function of temperature on approaching the critical point, are reported on Fig. 2 together with the theoretical estimate given by eq.(9). The parameters we used to compare theory and experiments are the ones pertinent for critical phenomena namely $\mathrm{D}=2.49, \tau=2.21$ and $v^{*}=0.71$ which characterizes the divergence of $\xi$ on approaching the critical point $\xi=\xi_{0}\left[\left(T-T_{c} / T_{c}\right]^{-v^{*}}\right.$, where the short range correlation length is $\xi_{0}=8.5 \AA$. We have adopted the value of the correlation length index renormalized according to the relation $v^{*}=v /(1-\alpha)$, where $\alpha$ is the critical index for the specific heat anomaly, which is appropriate for a threecomponent mixture $/ 9 /$.

It is clear that the experimental data are well described by eq.(9). In particular the crossover behavior which characterizes the transition from 
scattering by quasi-spherical monodispersed microemulsion droplets observed at low temperature and the critical behavior of the sample is rather well accounted for using a value of the droplet size given by $\mathrm{qR}_{1}=0.075$.

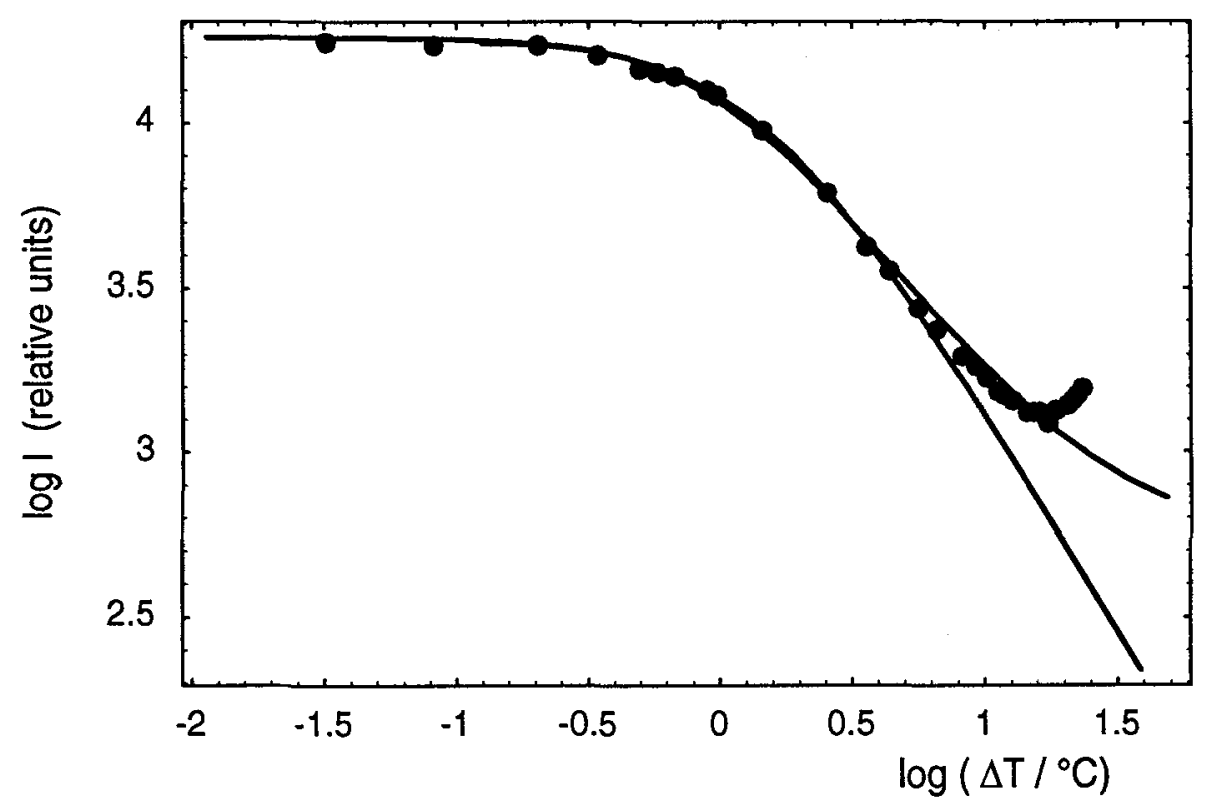

Fig. 2 The scattered light intensity as a function of the distance from the critical point $\Delta \mathrm{T}=\mathrm{T}_{\mathrm{c}}-\mathrm{T}$. The line bending upwards refer to $\mathrm{qR}_{1}=0.075$, the straight line to $\mathrm{qR}_{1}=0$. The value of $\xi_{0}=8.5 \AA$

Fig. 3 shows the measurements of the linewidth of the scattered radiation, performed along the critical concentration line as a function of temperature and at a scattering angle of $90^{\circ} / 9 /$. In this case too we observe a sudden change of behavior in the first cumulant which was attributed to a crossover from critical to single particle behavior. It is clear that the explicit inclusion of the finite particle size is able to reproduce the bending of the linewidth curve. Moreover the background need not to be added to the linewidth, since it is taken care of automatically by eq. (11).

As a conclusion we have presented a coherent picture that explains most of the experimental results obtained by different authors on static and dynamic light scattering studies in dense ternary AOT/Water/Decane microemulsions. The starting hypothesis is based on the polydispersed fractal nature of the aggregating microemulsion droplets close to the critical point. A theoretical model extending previous work on scattering from polydisperse fractal aggregates of colloidal system or sol-gel transition 
explains well the most typical aspects of the experimental observations, i.e. the increase of the scattered intensity at low values of the scattering wave vector, the crossover from a classic $q^{2}$ dependence of the first cumulant $\Gamma_{c}$ to a $\mathrm{q}^{3}$ dependence.

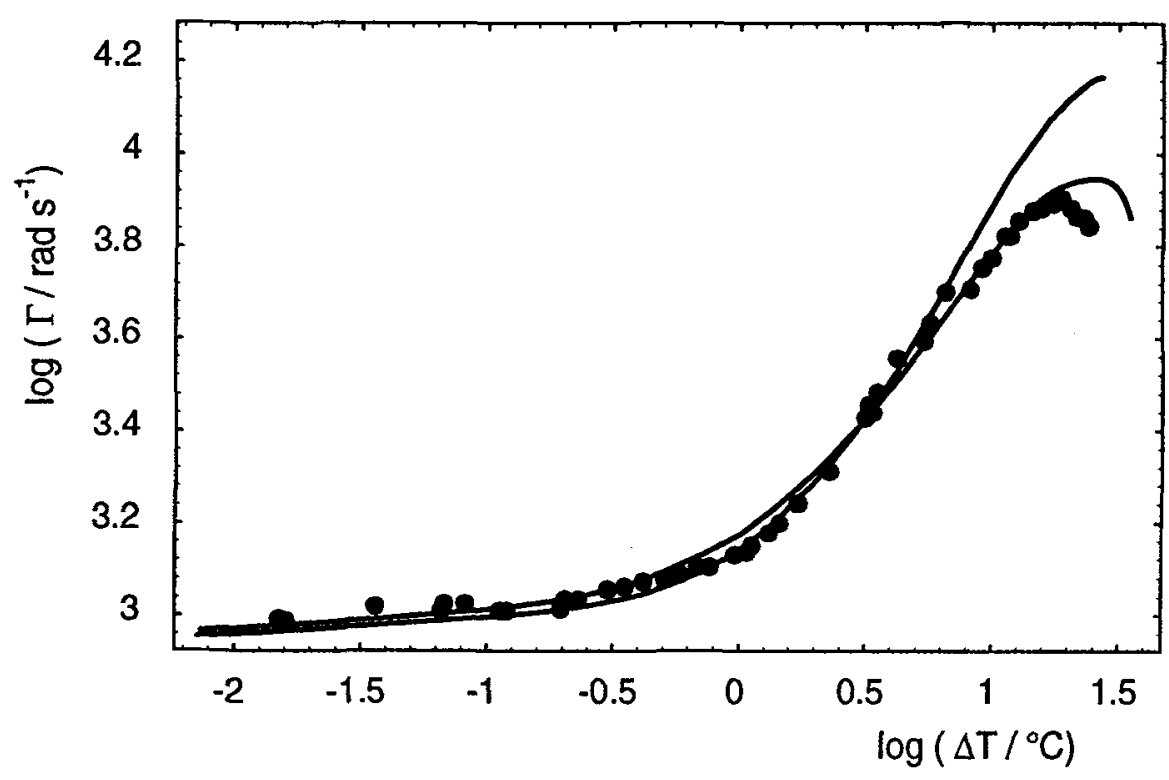

Fig. 3 The linewidth of the scattered light as a function of the distance from the critical point. The upper line refers to $\mathrm{qR}_{1}=0$, the lower one to $\mathrm{qR}_{1}=0.075$.

As a final comment, we want to stress that in the microemulsion system we are considering both critical and percolation phenomena coexist and can be described by scaling laws involving universal indices whose values vary only because of terms of the order of the index $\eta$. The physical quantities scale in terms of the variable $x=q \xi$ where $\xi$, the correlation length, is connected to the dimension of the average size cluster by $\xi=h R_{1} s^{1 / D}$, but varies as a function of composition and temperature with indices that are very different in the critical $(v \approx 0.63)$ and the percolative regions $(v \approx 0.88)$.

\section{Acknowledgments}

J.R. gratefully acknowledges the support and the hospitality of Roma I University La Sapienza during the time this work was completed. 


\section{References}

/1/ K. Kawasaki, in Phase Transitions and Critical Phenomena, edited by C. Domb and M.S. Green (Acad. Press., N.Y., 1976) Vol. 5A, p. 165.

/2/ P.C. Hohenberg, and B.I. Halperin, Rev. Mod. Phys. 49, 435 (1977).

/3/ See reviews by J.V. Sengers in Proceedings of the 1980 Cargèse Summer Institute on Phase Transitions, edited by H. Levy, J.C. Le Guillou, and J. Zinn-Justin (Acad. Press., N.Y., 1981), and D. Beysens, ibid.

/4/ H.C. Burstyn, J.V. Sengers, and P. Esfandary, Phys. Rev. A22, 282 (1980).

/5/ S.H. Chen, C.C. Lai, J. Rouch, and P. Tartaglia, Phys. Rev. A27, 1086 (1983).

/6/ P.G. Nilson, H. Wennerstrom, and B. Lindman, J. Phys. Chem. $\underline{88,} 309$ (1984).

/7/ J. Rouch, P. Tartaglia, A. Safouane, and S.H. Chen, Phys. Rev. A40, 2013 (1989).

/8/ J.E. Martin, and D.W. Schaefer, Phys. Rev. Lett. 53, 2457 (1984); J.E. Martin, and B.J. Ackerson, Phys. Rev. A31, 1180 (1985); J.E. Martin, and F. Leyuraz, Phys. Rev. A34, 2346 (1986); J.E. Martin, Phys. Rev. A36, 3415 (1987); J.E. Martin, J. Wilcoxon and J. Odinek, Phys. Rev. A43, 858 (1991).

19/ J. Rouch, A. Safouane, P. Tartaglia, and S.H. Chen, J. Chem. Phys. $\underline{90}$, 3756 (1989).

/10/ S.H. Chen, and J. Teixeira, Phys. Rev. Lett. 푸, 2583 (1986)

/11/ P. Tartaglia, J. Rouch, and S. H. Chen, Phys. Rev. A45, 7257 (1992).

/12/ A. Coniglio and W. Klein, J. Phys. A $\underline{13}, 2775$ (1980).

/13/ F. Perrot, P. Guenon and D. Beysens, in Universalities in Condensed Matter, R. Julien, L. Peliti, R. Rammal and N. Boccara eds,, p. 52 , Springer-Verlag Berlin 1988.

/14/ D. Stauffer Phys. Rep. 54, 1 (1979).

/15/ K. Kawasaki, Ann. Phys. (N.Y.). 61, 1 (1970).

/16/ R.A. Ferrell, Phys. Rev.Lett. 24, 1169 (1970), R. Perl, and R.A. Ferrel, Phys. Rev. A6, 2358 (1972). 